\title{
Begging test: uma avaliação da graduação da comunicação visual entre cães sem raça definida, cães de raça (Golden Retrievers) e humanos
}

\author{
Begging test: an evaluation of the degree of visual communication between mixed, pure bred dogs \\ (Golden Retrievers) and humans
}

Edilberto Nobrega Martinez ${ }^{\mathrm{I}}$ Vanner Boere ${ }^{\mathrm{II}}$

\section{RESUMO}

O begging test tem sido usado para testar a capacidade dos cães em interpretar a atenção humana. Pistas faciais, como o olhar e a posição da cabeça são os principais sinais na comunicação entre cães e humanos. Até o momento, a estrutura do begging test utilizado em pesquisas não permitiu verificar se há uma graduação na atenção. Desse modo, não é possível dizer se a cabeça e o olhar diretamente dirigidos (muita atenção) ao cão aumentam o desempenho em relação a somente a posição da cabeça, mas sem olhar diretamente (pouca atenção) ou cabeça plus olhar desviados (desatenção). Nesse sentido, um teste envolvendo 58 cães de raça pura (Golden Retrievers) e mista foi realizado, nas três condições de atenção, com homens e mulheres estranhos aos animais servindo de sujeitos experimentais. Resultados demonstraram que independente da raça, do sexo dos cães ou do sexo das pessoas, os cães erraram pouco na condição de desatenção. Por outro lado, o desempenho dos cães não diferiu entre as condições de muita atenção e de pouca atenção, mas foi significativamente superior do que na condição de desatenção. Em conclusão, os cães são bons interpretadores da atenção humana, mas se baseiam em pistas mais relacionadas à posição da cabeça do que o olhar. Assim, sugere-se que não há uma graduação na interpretação da atenção de humanos em cães. $O$ estímulo emocional gerado pelo olhar direto, sustentado entre animais sociais, pode explicar porque o olhar é evitado como pista da atenção humana em um teste com pessoas estranhas ao cão.

Palavras-chave: cão doméstico, ser humano, atenção, intenção, comunicação.

\section{ABSTRACT \\ The begging test has been used to test the ability of dogs to interprete human attention. Facial cues, such as gaze}

\begin{abstract}
and head position are the main signs in the communication between humans and dogs. Yet, the structure of the begging test used in research did not allow checking whether there are graduations of attention. Thus, it's not possible to say if the head and look directly driven (much attention) to the dog increases the performance in relation only to head position, but without looking directly (little attention) or head plus eyes diverted (inattention). Hence, a test using 58 mixed and pure bred dogs (Golden Retrievers) was performed, in three attention conditions, with men and women strangers to these animals, which served as experimental subjects. Results showed that regardless of race, dogs' gender of or people's gender, dogs have fewer errors on inattention condition Conversely, the performance of the dogs did not differ between the conditions of much attention and little attention, but was significantly higher than in the condition of inattention. In conclusion, dogs are good interpreters of human attention, but they make their choices based on cues related more to the head position than the look. Therefore, it is suggested that there isn't a interpretation degree of human attention in dogs. The emotional stimulus generated by the direct gaze, sustained among social animals, may explain why the gaze is averted as a cue of human attention in a test with strangers to the dog.
\end{abstract}

Key words: domestic dog, human being, attention, intention, communication.

\section{INTRODUÇÃO}

A comunicação entre canídeos é composta por um conjunto de sinais corporais, odores, vocalização e pelo olhar (FOX, 1971; BRADSHAW \& NOTT, 1995). O olhar pode mediar relações sociais,

'Programa de Pós-graduação em Biologia Animal, Universidade Federal de Viçosa (UFV), Viçosa, MG, Brasil.

"Departamento de Medicina e Enfermagem, UFV, 36570-000, Viçosa, MG, Brasil. E-mail: vanner.boere@ufv.br. Autor para correspondência 
como um meio de comunicação dentro de um grupo (EMERY et al., 1997). Entre canídeos, particularmente entre lobos (Canis lupus spp.) e cães domésticos (Canis lupus familiaris), o olhar parece ser importante para reforçar posições hierárquicas (FOX, 1971).

Em espécies sociais, a habilidade em detectar o direcionamento da intenção de outro indivíduo baseado em pistas comportamentais pode funcionar como uma importante prevenção de futuras ações do companheiro (VIRÁNYI et al., 2004). Em espécies sociais como lobos e cães domésticos, a percepção da intenção tem papel fundamental na interação comunicativa. O olhar também está envolvido nas pistas para interação na predação em grupo (BRADSHAW \& NOTT, 1995).

Em humanos, o olhar providencia o primeiro recurso para a informação da intenção e da atenção de outro. A orientação da cabeça e/ou corpo providencia pistas complementares para avaliar o foco de atenção de outro indivíduo (PERRET et al., 1992). Entre cães e humanos, a relação mediada pelo olhar e pela postura é bidirecional (MIKLÓSI, 2009). Tanto as pessoas podem perceber a atenção dos cães como estes podem interpretar a atenção dos humanos (MIKLÓSI et al., 2000).

Indivíduos de espécies diferentes podem interpretar sinais de perigo, de alarme e de emboscada (por exemplo, VALONE, 2007; WEBSTER et al., 2010). Os cães domésticos são capazes de seguir várias pistas humanas de atenção. $\mathrm{O}$ contraste entre olhos abertos ou fechados permite que os cães adquiram do olhar um componente importante para determinar o estado de atenção humana (CALL et al., 2003).

Cães domésticos são capazes de acertar onde um alimento está escondido pela interpretação da direção do olhar e da cabeça ou pelo apontamento com a mão de uma pessoa que indica o local (AGNETTAet al., 2000; HARE \& TOMASELLO, 1999; MIKLÓSI et al., 1998; SOPRONI et al., 2002). O sucesso é baseado no uso de pistas sociais, uma vez que explicações alternativas foram descartadas, incluindo uso de odor como pista para encontrar o alimento (HARE \& TOMASELLO, 1999; MCKINLEY \& SAMBROOK, 2000; SZETEI et al., 2003). Cães são capazes de devolver uma bola na brincadeira de buscar para os proprietários que mantém maior tempo de contato visual (GÁCSI et al., 2004).

Em um teste de comunicação (begging test) envolvendo pessoas, os cães escolhem um indivíduo para pedir comida baseado em pistas faciais normalmente associadas com a atenção (GÁCSI et al., 2004). O begging test é particularmente interessante $\mathrm{e}$ simples, podendo explicar como os cães podem melhor aprender e responder à interação com pessoas. Basicamente, o teste consiste em se colocar um cão, seguro por uma guia, a poucos metros frente a duas pessoas que estão segurando um sanduíche, simulando comê-lo. Uma das pessoas olha fixamente para o cão durante um minuto enquanto a outra vira a cabeça ou está com os olhos vendados. Após ser solto da guia o cão se aproxima, mais frequentemente, da pessoa, entre as duas, que o havia observado previamente. $\mathrm{O}$ cão pode tocar com o focinho, tentar atingir o sanduíche ou apenas permanecer olhando fixamente a pessoa. GÁCSI et al. (2004) aplicaram o begging test para concluir que os cães são capazes de interpretar a atenção humana pelo olhar e postura da cabeça. Contudo, o estudo de GÁCSI et al. (2004) não deixa claro se cães podem compreender apenas pelo olhar ou se a postura da cabeça também influenciaria a decisão de pedir comida à pessoa que com ele interage.

A interpretação de sinais sociais de humanos pelos cães, tais como atenção e reciprocidade, pode estar em um espectro gradativo ou simplesmente é um parâmetro dicotômico? Isto é, podem os cães avaliar e tomar decisões na intensidade da atenção e da reciprocidade expressa por humanos? Ou simplesmente a decisão é de atenção/reciprocidade ou não-atenção/não-reciprocidade? Os estudos até o momento sobre a comunicação dentre cães e humanos não abordam essa questão, segundo a revisão que realizamos. Os estudos têm sido direcionados para determinar uma resposta erro-acerto. Em uma interação social com vários atores, seria desvantajoso um dentre eles permanecer insistindo numa reciprocidade com outro ator não-atento ou não-agente. Em outras palavras, espera-se que, assim como humanos, haja uma intensidade de reciprocidade em cães se comunicando com humanos.

A domesticação dos cães, um processo que pode ter ocorrido em torno de 14000 anos atrás (CLUTTON-BROCK, 1995) levou ao refinamento na seleção de caracteres físicos e comportamentais de cães, caracterizando raças com atributos especiais para determinadas funções. Algumas raças parecem desempenhar melhor algumas tarefas do que outras. Cães mestiços, entretanto, por cruzamentos sucessivos, sofrem uma gradual regressão a um estado supostamente primitivo em caracteres fenotípicos (CLUTTON-BROCK, 1995). Apesar dessa especialização na capacidade de desempenhar tarefas conforme selecionado, os cães essencialmente desempenham um papel social de estreita comunicação com humanos. Essa característica parece ser inerente ao $\boldsymbol{C}$. lupus familiaris e a seleção por raças não teria influenciado o desempenho social na comunicação com 
humanos. Portanto, não está claro se raças puras ou cães mestiços possuem maior capacidade de interpretar a atenção humana.

Neste estudo, avaliou-se a graduação da capacidade do cão em reconhecer, analisar e tomar decisões baseado no nível de atenção humana a ele direcionada. Nossa hipótese é que há uma diferença significativa na interpretação dos cães do grau de atenção de uma pessoa. Isto é, a maioria dos cães pede alimento para um sujeito que se comunica com a direção do olhar, e não para uma pessoa que desvia o olhar ou que vira a cabeça para o lado. No caso de apenas desviar o olhar e virar a cabeça para o lado, os cães são mais capazes de pedir alimento para aquele que desempenha a primeira ação. Adicionalmente, averiguou-se a relação do desempenho no begging test e possíveis diferenças entre uma raça pura e cães mestiços.

\section{MATERIAL E MÉTODOS}

Realizou-se o begging test adaptado do estudo de GÁCSI et al. (2004) com três repetições consecutivas, em 43 cães mestiços pertencentes a abrigos e 15 Golden Retrievers pertencentes a canis especializados. Os experimentos ocorreram em ambiente familiar aos cães. Os cães estavam em jejum por no mínimo quatro horas antes do início dos testes.

Três pessoas do sexo masculino e três do sexo feminino, com tipo físico semelhante, sem contato prévio com os animais, fizeram parte do teste como sujeitos de atenção. As pessoas participantes foram orientadas a não utilizar perfumes, desodorantes com perfume e nem lavar a cabeça com produtos para cabelo 24 horas antes do início de cada etapa do experimento. Diferentes cães foram utilizados para homens e mulheres para evitar habituação ao sujeito pelos animais. As pessoas trajavam vestimentas similares, com a mesma cor. O local para desempenhar a avaliação constituíase de uma área externa (varanda) com aproximadamente $15 \mathrm{~m}^{2}$, despojado de móveis, com exceção de três cadeiras do mesmo modelo e cor para os testes.

Os participantes sentaram-se em cadeiras, usando óculos escuros e com a cabeça voltada para frente, sem se mover. A seguir, o cão foi introduzido no ambiente pelo manipulador, desprovido de guia, a uma distância de três metros dos participantes. O cão, ao ingressar na área, poderia se locomover livremente, assim como se aproximar dos participantes, mas sem interação como afago, gestos, olhares e falar ao animal. Ao término de um minuto, o pesquisador dava um pedaço de sanduíche e retirava o cão do ambiente, para dar início dentro de 30 s à sequência de três tentativas do begging test.
$\mathrm{Na}$ sequência de três tentativas, as três pessoas (denominadas de A, B e C) com sanduíche nas mãos, ficaram alinhadas e sentadas a $0.75 \mathrm{~m}$ de distância uma da outra; a pessoa do meio encontravase a três metros de distância do ponto de retenção do cão antes da soltura. Os participantes seguraram o sanduíche com ambas as mãos na altura do tórax e mastigaram um pedaço durante cada tentativa. Quando cada pessoa encontrava-se sentada e com a disposição corporal específica pré-determinada, deu-se início a primeira tentativa, seguida de outras duas.

Durante trinta segundos, o manipulador manteve o cão seguro no ponto de partida situado à frente dos participantes, dando-lhe a oportunidade de avaliar os participantes. Três disposições de contato foram sugeridas: o participante poderia manter a cabeça direcionada ao cão com contato visual; ou poderia manter a cabeça direcionada ao cão, porém com o olhar direcionado para o teto; ou, ainda, direcionar a cabeça para um dos lados aleatoriamente escolhido, impossibilitando qualquer contato visual com o cão (ver pictograma na figura 1). Passados os trinta segundos, o manipulador soltava o cão, que poderia se aproximar da pessoa A, B ou C para pedir comida. Feita a escolha, ou passado 60s, o operador retirava gentilmente o cão da sala. Ao término de cada etapa, o cão era colocado em uma sala adjacente fechada, por trinta segundos, para que os participantes pudessem trocar de posições e/ou disposição corporal, de forma aleatoriamente escolhida.

Cada escolha do cão foi graduada com três escores possíveis. Escore igual a 1,0 a cada tentativa que o cão escolhesse o participante com contato visual; escore igual a 0,5 para a escolha da pessoa com o direcionamento da cabeça para o cão, porém com olhar para o teto; e um escore igual a zero no caso de o cão escolher a pessoa com a cabeça virada para o lado. Tentativas em que o cão não se aproximava dos participantes não foram pontuadas.

Os cães foram analisados em relação à condição de pontuação individualmente e foi atribuído um escore médio percentual entre as tentativas. Os cães foram classificados como bons interpretadores se o escore final fosse superior ou igual a 2, como intermediários se a pontuação fosse menor que 2 e maior ou igual a 1; e como interpretadores ruins quando o desempenho fosse abaixo de 1. Esses escores permitiram classificar os cães em três grupos representados em percentagens.

Adotando o desempenho dos cães como fator randômico, realizaram-se testes não-paramétricos, teste Mann Whitney, para verificar possíveis diferenças quanto à raça (Golden Retriever vs. sem-raça-definida), 


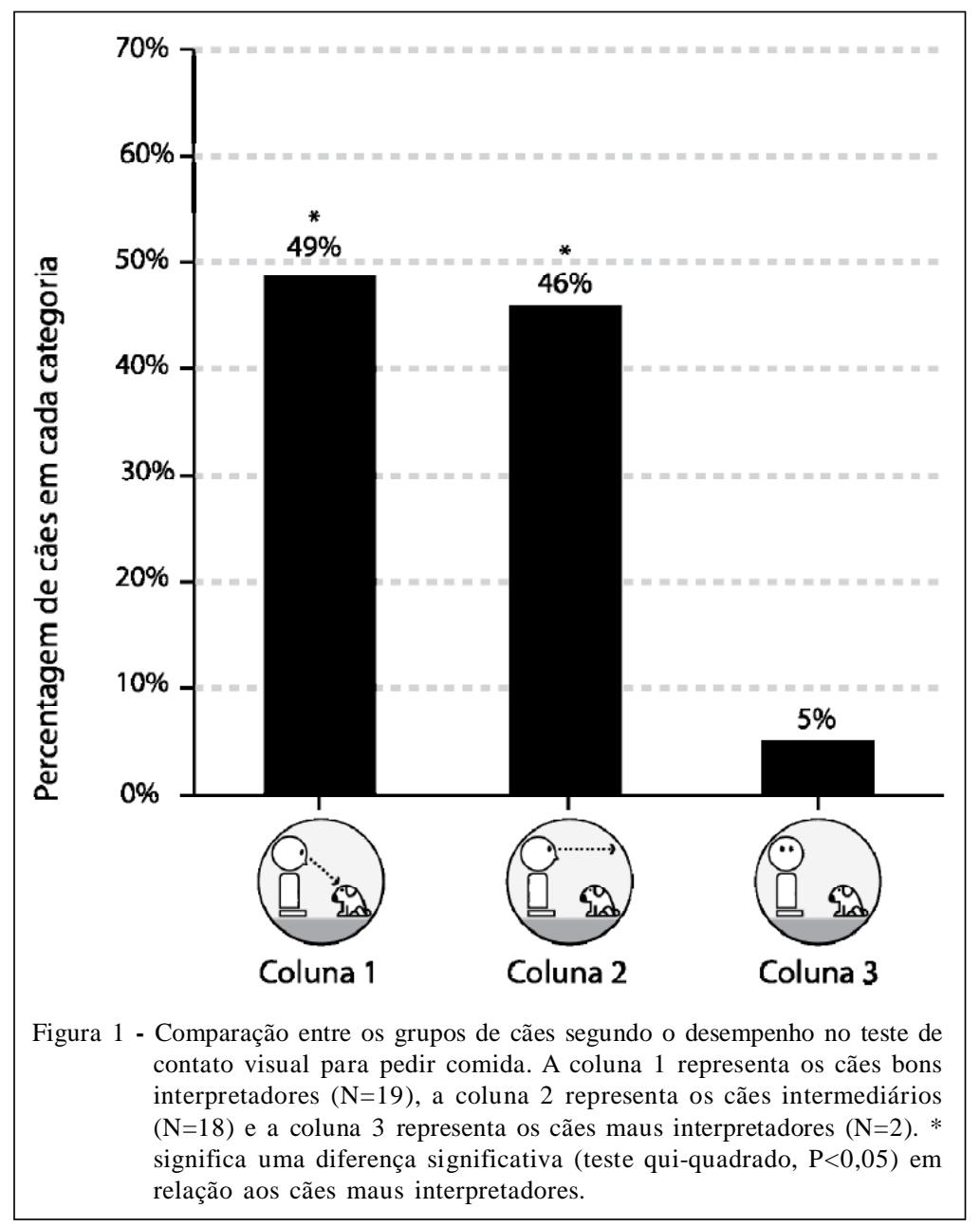

o sexo dos cães, o sexo das pessoas e o pareamento sexo do cão plus sexo das pessoas. Para comparar os cães por desempenho, utilizaram-se testes quiquadrados. Os testes estatísticos tiveram distribuição bicaudal, com a menor ou igual a 0,05 .

\section{RESULTADOS}

Dos 58 cães, 39 completaram pelo menos uma das tentativas, totalizando 89 tentativas. Não houve diferença de desempenho quando se comparou os cães por raça (Golden Retriever, $\mathrm{n}=12$; mestiços, $\mathrm{n}=27$; teste Mann-Whitney, $\mathrm{Z}=-0,267, \mathrm{P}=0,79$ ) e por sexo (machos, $n=12$, fêmeas, $n=27$; teste MannWhitney, $\mathrm{Z}=-0,158, \mathrm{P}=0,89)$. Nem os cães tiveram performances diferentes quando testados com homens ou com mulheres (homens, $n=12$, mulheres, $n=27$; $\mathrm{P}>0,05)$. Ocorreu uma diferença significativa entre ambas as categorias por desempenho, os cães bons interpretadores e os cães interpretadores intermediários (Figura 1), em relação aos cães maus interpretadores
$(\mathrm{X} 2=14,0 ; \mathrm{g} .1 .=2 ; \mathrm{P}<0,01)$. Não ocorreu diferença $(\mathrm{P}>0,05)$ no percentual de cães bons interpretadores $(49 \%)$ e de cães intermediários $(46 \%)(\mathrm{X} 2=0,027 ; \mathrm{P}=0,87)$.

\section{DISCUSSÃO}

Poucos cães erraram ao escolher pessoas que estavam olhando para o outro lado. A maior parte dos acertos ocorreu nas duas situações: quando os olhos fitavam o cão ou quando os olhos fitavam o teto; em ambos os casos, a cabeça estava direcionada para o cão.

Os resultados do presente estudo confirmam os achados do begging test em cães conduzido por GÁCSI et al. (2004). Os cães possuem a capacidade de interpretar a atenção do ser humano em pistas faciais ou pela posição da cabeça. Contudo, em nossa abordagem, não encontramos uma gradação da resposta conforme a direção do olhar ou a posição da cabeça. Considerando que, na condição de olhar direto e olhar para cima, a cabeça encontrava-se na posição 
dirigida (apontada) para o cão, os nossos resultados sugerem que os cães se utilizam da posição da cabeça para a interpretação da atenção humana, mesmo que a direção do olhar possa indicar a falta de atenção e aumentar o potencial de um erro de comunicação.

O olhar é importante naquelas espécies que possuem sinais faciais emitidos por complexos músculos que podem mover a região orbicular e compor sinais com várias estruturas da face (EMERY, 2000). Essa capacidade de interpretação apenas pelo olhar pode ser uma característica exclusiva de primatas, particularmente antropóides (EMERY, 2000). Outros vertebrados sociais se utilizam de vários outros sinais corporais para interpretar a atenção de outros animais. Para animais caçadores cooperativos, a atenção pode ser melhor interpretada pela posição da cabeça e outras partes do corpo, compondo uma mensagem da disposição do animal (EMERY, 2000). A posição da cabeça indicaria essa disposição de atenção entre dois animais como os cães. Portanto, os cães podem não ter desenvolvido a priori a capacidade de interpretar a atenção humana apenas pelo olhar. Não significa que os cães não possuam a capacidade de interpretar a atenção humana pelo olhar; essa capacidade pode ser um processo de aprendizado na socialização dos cães com os humanos.

A ambiguidade na interpretação dos sinais (olhar vs posição da cabeça) pode ter um significado mais complexo do que apenas a atenção correspondida. Alguns estudos sugerem que o olhar fixo pode funcionar como uma pista social na interação humano e cão, enquanto a duração do olhar parece estar relacionada com o ritual de agressão e dominância. $\mathrm{O}$ olhar dirigido aos olhos de um oponente acarreta uma intensa ativação da amígdala, uma região do córtex emocional responsável por mediar emoções de defesa (WHALEN et al., 2004). Desviar o olhar indica subordinação na interação visual entre dois cães (BRADSHAW \& NOTT, 1995). A escolha dos cães por pessoas que estavam olhando para cima, mas com a cabeça dirigida para frente, pode ser uma estratégia de evitar o contato direto nos olhos de um estranho (o participante), evitando uma possível interação agonística. Dessa forma, os cães no presente estudo, estariam tentando estabelecer uma comunicação, mas sem parecer estar desafiando a pessoa.

O sexo dos participantes parece não influenciar na capacidade dos cães de interpretar sinais de atenção. Embora existam estudos sugerindo que cães e particularmente cadelas (WELLS \& HEPPER, 1999) se comportam mais amistosamente com mulheres do que com os homens, os sinais dessa preferência (se houver) não parecem influenciar na capacidade de interpretar a atenção humana pelo olhar ou pela posição da cabeça unicamente.

Por fim, a capacidade de cães interpretarem a atenção pela posição da cabeça de uma pessoa não parece estar condicionada a uma raça de cães buscadores de caça (Golden Retriever) ou cães mestiços. Os milhares de anos de seleção na domesticação dos cães parecem não ter interferido na capacidade de interpretar a atenção humana. Contudo, outras raças selecionadas para outras funções necessitam ser investigadas para uma assertiva mais abrangente.

\section{CONCLUSÃO}

Baseado apenas pelo olhar, não há uma graduação da atenção em cães domésticos. A alta eficiência de acerto no begging test sugere que em cães domésticos a posição da cabeça parece ser uma pista importante para a interpretação da atenção humana, independentemente de sexo em mestiços ou golden retrievers.

\section{AGRADECIMENTOS}

Agradecemos às nossas famílias e às pessoas e animais participantes dos procedimentos de campo, que tornaram a realização do projeto viável.

\section{REFERÊNCIAS}

AGNETTA, B. et al. Cues to food location that domestic dogs (Canis familiaris) of different ages do and do not use. Animal Cognition, v.3, p.107-112, 2000. Disponível em: <http:// www.springerlink.com/content/192fyae 2 qkydfhq3/>. Acesso em: 19 set. 2009. doi: 10.1007/s100710000070.

BRADSHAW, J.W.S.; NOTT, H.M.R. Social and communication behavior of companion dogs. In: SERPELL, J. The domestic dog, its evolution, behavior and interactions with people. Cambridge: Cambridge University, 1995. p.115-130.

CALL, J. et al. Domestic dogs (Canis familiaris) are sensitive to the attentional state of humans. Journal of Comparative Psychology, v.117, p.257-263, 2003. Disponível em: <http:/ /psycnet.apa.org/journals/com/117/3/257/>. Acesso em: 19 set. 2009. doi: 10.1037/0735-7036.117.3.257 .

CLUTTON-BROCK, J. Domestication and evolution. In: SERPELL, J. The domestic dog, its evolution, behaviour and interactions with people. Cambridge: Cambridge University, 1995. p.7-20.

EMERY, N.J. The eyes have it: the neuroethology, function and evolution of social gaze. Neuroscience and Biobehavioral Reviews, v.24, p.581-604, 2000. Disponível em: <http:// www.sciencedirect.com/science?_ob=MImg\&_imagekey=B6T0J$40 X N W 1 G-1-P \& \_c d i=4864 \& \_$user $=687355 \&$ \& pii $=$ S0 149 $763400000257 \&$ _orig =search\&_coverDate $=08 \%$ 2F31\%2F2000\&_sk=999759993\&view =c\&wchp=dGLbVlb- 
zSkzS\&md5=0fed 81 f0d00807c3224c4d67 1d4ac0d9\&ie $=/$ sdarticle.pdf $>$. Acesso em: 10 set. 2009. doi: 10.1016/S01497634(00)00025-7.

FOX, M.W. Behaviour of wolves, dogs and related canids. London: Jonathan Cape, 1971. 214p.

GÁCSI, M. et al. Are readers of our face readers of our minds? Dogs (Canis familiaris) show situation-dependent recognition of human's attention. Animal Cognition, v.7, p.144-153, 2004. Disponível em: <http://www.springerlink.com/content/ c2d12pyxyugg4ybp>. Acesso em: 13 out. 2009. doi: 10.1007/ s10071-003-0205-8.

HARE, B.; TOMASELLO, M. Domestic dogs (Canis familiaris) use human and conspecific social cues to locate hidden food. Journal of Comparative Psychology, v.113, p.173-177, 1999. Disponível em: <http://psycnet.apa.org/journals/com/ 113/2/173>. Acesso em: 19 set. 2009. doi: 10.1037/07357036.113.2.173

MCKINLEY, J.; SAMBROOK, T.D. Use of human-given cues by domestic dogs (Canis familiaris) and horses (Equus caballus). Animal Cognition, v.3, p.13-22, 2000. Disponível em: <http://www.springerlink.com/content/ur42nvvgq6rekf64>. Acesso em: 13 out. 2009. doi: 10.1007/s100710050046.

MIKLÓSI, A. et al. Use of experimenter- given cues in dogs. Animal Cognition, v.1, p.113-121, 1998. Disponível em: <http://www.springerlink.com/content/ebwred7k0kvx53bw>. Acesso em: 19 set. 2009. doi: 10.1007/s100710050016.

MIKLÓSI, A. et al. Intentional behaviour in dog-human communication: an experimental analysis of 'showing' behaviour in the dog. Animal Cognition, v.3, p.159-166, 2000. Disponível em: <http://www.springerlink.com/content/ m0c2fah0685tbhaj>. Acesso em: 19 set. 2009. doi: 10.1007/ s100710000072.

MIKLÓSI, A. Evolutionary approach to communication between humans and dogs. Veterinary Research Communications, v.33, p.53-59, 2009. Disponível em: <http:// www.springerlink.com/content/x1p3w5543u3105t2>. Acesso em: 03 maio, 2010. doi: 10.1007/s11259-009-9248-x.

PERRET, D.I. et al. Organization and fuction of cells responsive to faces in the temporal córtex. Philosophical Transactions of the Royal Society. Biological Sciences, v.335, p.23-30, 1992. Disponível em: <http://www.jstor.org/pss/55471>. Acesso em: 29 nov. 2009.

SOPRONI, K. et al. Dog's (Canis familiaris) responsiveness to human pointing gestures. Journal of Comparative Psychology, v.116, p.27-34, 2002. Disponível em: <http:// psycnet.apa.org/index.cfm?fa $=$ fulltext.printArticle $\& j c$ ode $=$ com $\&$ vol $=116 \&$ issue $=1 \&$ format $=$ html\&page $=27 \&$ language $=$ eng $>$. Acesso em: 19 set. 2009. doi: 10.1037/0735-7036.116.1.27.

SZETEI, V. et al. When dogs seem to lose their nose: an investigation on the use of visual and olfactory cues in communicative context between dog and owner. Animal Behaviour Science, v.83, p.141152, 2003. Disponível em: <http://www.sciencedirect.com/ science?_ob=ArticleURL\&_udi=B 6T 48-48TMFB 5$5 \&$ \& u ser $=687355 \&$ \& c over D a t e $=09 \% 2$ F 05 $\% 2 \mathrm{~F} 2003 \&$ \&doc $=1 \&$ \& fmt $=$ high\&_orig $=$ search \& sort $=$ d\&_docanchor $=\&$ view $=c \&$ csearchStrId $=1336833095 \&$ _rerunOrigin $=$ scholar.google $\& \_$acct $=C 000037918 \& \_$version $=1 \&$ _urlVersion $=0 \& \_$userid $=687355 \mathrm{dmd5}=6 \mathrm{c} 4 \mathrm{~d} 729 \mathrm{dfcbdfOfada} 7984 \mathrm{e} 07 \mathrm{~b} 7 \mathrm{ab} 4 \mathrm{lb}>$. Acesso em: 19 set. 2009. doi: 10.1016/S0168-1591(03)00114-X.

VALONE, T.J. From eavesdropping on performance to copying the behavior of others: a review of public information use. Behavioural Ecology Sociobiology, v.62, p.1-14, 2007. Disponível em: <http://www.springerlink.com/content/ 26uw538855555432>. Acesso em: 30 nov. 2009. doi: 10.1007/ s00265-007-0439-6.

VIRÁNYI, Z.S. et al. Dogs respond appropriately to cues of humans' attentional focus. Behavioral Processes, v.66, p.161-172, 2004. Disponível em: <http:// w w w . s c i e $n$ c e d i r e c t . c o m / science?_ob=ArticleURL\&_udi=B 6T 2J -4BVRXJC$1 \&$ _user $=687355 \&$ _coverDate $=05 \% 2 \mathrm{~F} 31 \% 2 \mathrm{~F} 2004 \&$ \&doc $=1 \& \_f m t=h i g h \&$ orig $=$ search\&_sort $=d \& \_$docanchor $=\&$ view $=c \&$ \&searchStrId $=1336834629 \&$ _rerunOrigin $=$ scholar.google\&_acct $=$ C $000037918 \&$ \& version $=1 \&$ \& ur 1 Versio n $=0 \&$ _ u se rid $=687355 \& \mathrm{~m}$ d5=98fbfd1459ab6f5fb14b4fd6df832235>. Acesso em: $10 \mathrm{jul}$. 2009. doi: 10.1016/j.beproc.2004.01.012.

WHALEN, P.J. et al. Human amygdala responsivity to masked fearful eye whites. Science, v.306, n.5704, p.2061, 2004. Disponível em: <http://www.sciencemag.org/cgi/content/full/306/ 5704/2061 ?ijkey $=$ FdvOhgvRZa $7 w Q \&$ keytype $=$ ref\&siteid $=$ sci $>$. Acesso em: 10 set. 2009. doi: 10.1126/science.1103617.

WEBSTER, H. et al. Eavesdropping and risk assessment between lions, spotted hyenas and African wild dogs. Ethology, v.116, p.233239, 2010. Disponível em: <http://www3.interscience.wiley.com/ cgi-bin/fulltext/123261260/PDFSTART>. Acesso em: 02 maio, 2010. doi: 10.1111/j.1439-0310.2009.01729.x.

WELLS, D.L.; HEPPER, P.G. Male and female dogs respond differently to men and women. Animal Behaviour Science, v.61, p.341-349, 1999. Disponível em: <http:// www.sciencedirect.com/science?_ob=MImg\&_imagekey=B6T483 VCK40B-8-7\&_cdi $=4968 \&$ \&user $=687355 \&$ _pii $=$ S 0 $168159198002020 \&$ orig $=$ search \&_coverDate $=01 \%$ 2F28\%2F1999\&_sk=999389995\&view $=c \& w c h p=d G L z V t b-$ zSkWb\&md5 $=\mathrm{d} 42628$ ec535f386c 89 fab34b0fef6de0 \&ie $=/$ sdarticle.pdf>. Acesso em: 19 set. 2009. doi: 10.1016/S01681591(98)00202-0. 